\title{
Predictors of oncological outcomes in T1G3 patients treated with BCG who undergo radical cystectomy
}

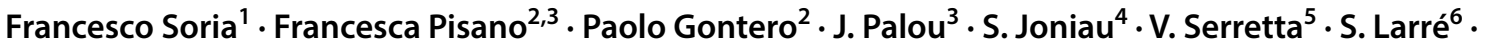

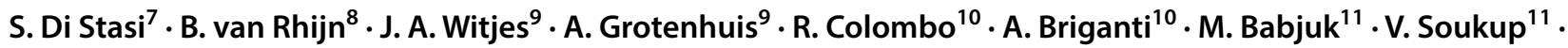

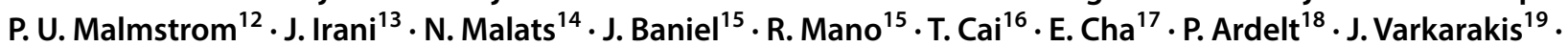 \\ R. Bartoletti ${ }^{20}$. G. Dalbagni ${ }^{21}$. S. F. Shariat ${ }^{1} \cdot$ E. Xylinas $^{22} \cdot$ R. J. Karnes ${ }^{23} \cdot$ R. Sylvester ${ }^{24}$
}

Received: 1 March 2018 / Accepted: 13 August 2018 / Published online: 31 August 2018

(c) Springer-Verlag GmbH Germany, part of Springer Nature 2018

\begin{abstract}
Purpose To evaluate the oncological impact of postponing radical cystectomy (RC) to allow further conservative therapies prior to progression in a large multicentre retrospective cohort of T1-HG/G3 patients initially treated with BCG.

Methods According to the time of RC, the population was divided into 3 groups: patients who did not progress to muscleinvasive disease, patients who progressed before radical cystectomy and patients who experienced progression at the time of radical cystectomy. Clinical and pathological outcomes were compared across the three groups.

Results Of 2451 patients, 509 (20.8\%) underwent RC. Patients with tumors $>3 \mathrm{~cm}$ or with CIS had earlier cystectomies $(\mathrm{HR}=1.79, p=0.001$ and $\mathrm{HR}=1.53, p=0.02$, respectively). Patients with tumors $>3 \mathrm{~cm}$, multiple tumors or CIS had earlier T3/T4 or $\mathrm{N}+$ cystectomies. In patients who progressed, the timing of cystectomy did not affect the risk of T3/T4 or $\mathrm{N}+$ disease at RC. Patients with T3/T4 or N + disease at RC had a shorter disease-specific survival $(\mathrm{HR}=4.38, p<0.001)$, as did patients with CIS at cystectomy $(\mathrm{HR}=2.39, p<0.001)$. Patients who progressed prior to cystectomy had a shorter disease-specific survival than patients for whom progression was only detected at cystectomy $(\mathrm{HR}=0.58, p=0.024)$

Conclusions Patients treated with RC before experiencing progression to muscle-invasive disease harbor better oncological and survival outcomes compared to those who progressed before RC and to those upstaged at surgery. Tumor size and concomitant CIS at diagnosis are the main predictors of surgical treatment while tumor size, CIS and tumor multiplicity are associated with extravesical disease at surgery.
\end{abstract}

Keywords Bladder cancer · High risk · T1G3 - Cystectomy · Outcomes · Extravesical disease

\section{Introduction}

The optimal management of primary T1 bladder cancer (BCa) remains a challenge, mainly due to its highly variable behavior [1]. While the majority of T1G3 BCa has a clinical response to intravesical Bacillus Calmette-Guerin (BCG) therapy resulting in a lowering of the risk of disease progression, a subgroup of patients will experience early progression to muscle-invasive $\mathrm{BCa}$ [1-3]. This accounts for

Francesco Soria and Francesca Pisano contributed equally to this work.

Francesca Pisano

francescapisano85@gmail.com

Extended author information available on the last page of the article the $10-30 \%$ rate of cancer-specific mortality in T1G3 BCa $[4,5]$. Several investigators have tried to identify individual risk factors for disease worsening such as patient age, concomitant carcinoma in situ (CIS), multifocality, large tumors $(>3 \mathrm{~cm})$, lymphovascular invasion (LVI) and histological variants [1, 2, 6]. Moreover, despite an increasing uptake of repeated transurethral resection (re-TUR) as part of routine clinical practice in high-risk patients, pathological upstaging at the time of $\mathrm{RC}$ is found in about $30 \%$ of patients [7, 8]. Therefore, some researchers have advocated an immediate $\mathrm{RC}$ in patients with poor prognostic factors [9]. However, some patients with T1G3 BCa without unfavorable features will also require $\mathrm{RC}$ after an initial conservative treatment such as BCG due to the development of multiple recurrences or progression to muscle-invasive disease [10]. It has been suggested that this delay in performing radical surgery has 
a negative impact on recurrence-free (RFS), cancer-specific (CSS) and overall survival (OS) rates [11].

The optimal timing of RC remains the main challenge in T1 high-grade patients treated with BCG. Moreover, identification of the patients who are likely to benefit from an early $\mathrm{RC}$, while deferring a morbid surgery in those who do not need it, is essential. The aim of our study is to evaluate the oncological impact of postponing $\mathrm{RC}$ to allow further conservative therapies prior to progression and to better define the role of early RC in this category of patients.

\section{Methods}

\section{Study population}

The study design and patient selection criteria have been previously reported by Gontero et al. [2]. Briefly, patients with primary T1G3 (WHO 1973)/T1 high-grade (HG) (ISUP 1998/WHO 2004) tumors or secondary T1G3/HG disease in a previously BCG naïve non-T1G3/HG NMIBC tumor were included in this multicenter retrospective study. All patients received at least a full induction course of BCG between 1990 and 2011. Patients who underwent radical cystectomy were specifically retained for the purpose of this study and included in the analyses.

\section{Data collection}

The following patient and tumor characteristics were collected: age, gender, smoking history and intensity, exposure to chemical compounds, tumor status (primary or recurrent), previous intravesical chemotherapy, tumor size $(<$ versus $>3 \mathrm{~cm}$ ), tumor focality (solitary versus multiple), presence of concomitant CIS, prostatic urethra involvement with or without stromal invasion, the presence of muscle in the primary tissue specimen, reTUR and pathology at reTUR, BCG dose and total number of instillations. For patients undergoing radical surgery, the following data were collected: time to radical cystectomy, disease status before cystectomy, stage and nodal status at cystectomy, cancerspecific and overall mortality.

\section{Statistical analysis}

Primary end-point of the study was to evaluate the impact of timing of cystectomy in T1G3 patients treated with BCG on both pathologic stage at cystectomy and disease-specific survival outcomes. Cystectomy was classified into 3 groups based on its timing relative to progression to muscle-invasive disease: patients undergoing radical cystectomy who did not progress to muscle-invasive disease, patients who progressed to muscle-invasive disease before radical cystectomy and patients who experienced progression to muscle-invasive disease at the time of radical cystectomy. The secondary end-point was to identify prognostic factors for time to radical cystectomy and time to extravesical disease at radical cystectomy (T3/T4 disease and/or presence of $\mathrm{N}+$ disease at radical cystectomy). Univariable and multivariable Cox regression analyses (including age, gender, the number of tumors, tumor size, concomitant CIS, reTUR and the presence of muscle, and maintenance BCG) were performed to identify factors predicting time to radical cystectomy and time to extravesical disease at radical cystectomy, which also took into account the timing of progression relative to cystectomy. Cancer-specific survival after radical cystectomy was also compared based on disease status at cystectomy and the timing of progression relative to cystectomy. Depending on the analysis, times to events were calculated using the Kaplan-Meier estimate taking the date of starting BCG or the date of cystectomy as time zero. Patients without an event or death prior to the event were censored at the last date of follow-up. Categorical variables were reported as absolute numbers and percentages and were compared using a Chi-square test. Statistical analyses were performed using STATA 12 (Stata Corp., College Station, TX, USA). All tests were two-sided and $p<0.05$ was considered as statistically significant.

\section{Results}

Five-hundred and nine (20.8\%) of the 2451 available patients underwent radical cystectomy (RC). Baseline patient and disease characteristics are reported in Table 1. Patients were more likely to have undergone $\mathrm{RC}$ if they had tumors $\geq 3 \mathrm{~cm}$ ( $25 \%$ versus $15 \%)$, multifocal tumors (26\% versus $15 \%$ ), concomitant CIS (27\% vs 19\%), no muscle in the TUR (29\% vs $20 \%$ ), T1 tumor at reTUR ( $43 \%$ vs $24 \%$ for Ta and $16 \%$ for $\mathrm{T} 0)$ or did not receive maintenance BCG (24\% versus $15 \%)$.

Table 2 presents disease characteristics at RC and survival according to the timing of RC. Radical surgery was performed without sign of progression in 172 (34\%) of the cases, while progression to muscle-invasive disease was observed before cystectomy and only at final cystectomy pathology in $226(44 \%)$ and $111(22 \%)$ of the patients, respectively. The timing of cystectomy was mainly influenced by T1G3 tumor size and the presence of concomitant CIS (Fig. 1).

A multivariable Cox regression analysis showed that patients with tumors $\geq 3 \mathrm{~cm}$ or with CIS have earlier cystectomies $(\mathrm{HR}=1.75,95 \%$ CI $1.40-2.19, p<0.001$ and $\mathrm{HR}=1.64,95 \%$ CI 1.30-2.07, $p<0.001$, respectively).

Among the 337 patients who experienced progression (either before or at surgery), $153(45 \%)$ had locally 
Table 1 Patient and disease characteristics of the cohort of 2451 T1G3 bladder cancer patients treated with BCG

\begin{tabular}{|c|c|c|c|c|}
\hline \multirow[t]{2}{*}{ Variables } & \multirow[t]{2}{*}{ Total } & \multicolumn{2}{|c|}{ Radical cystectomy } & \multirow[t]{2}{*}{$p$ value } \\
\hline & & No & Yes & \\
\hline Number of patients & 2451 & 1942 & 509 & \\
\hline $\begin{array}{l}\text { Median age (IQR), } \\
\text { years }\end{array}$ & $68(60-74)$ & $68(60-75)$ & $67(61-73)$ & \\
\hline \multicolumn{4}{|l|}{ Gender, $n(\%)$} & 0.20 \\
\hline Female & 439 (18) & $338(77)$ & $101(23)$ & \\
\hline Male & $2012(72)$ & $1604(80)$ & $408(20)$ & \\
\hline \multicolumn{4}{|c|}{ Smoking status, $n(\%)$} & 0.38 \\
\hline Never smoker & 469 (19) & $385(82)$ & $84(18)$ & \\
\hline Former smoker & $618(25)$ & $490(79)$ & $128(21)$ & \\
\hline Current smoker & 465 (19) & $382(88)$ & $83(18)$ & \\
\hline \multicolumn{4}{|l|}{ Tumor status, $n(\%)$} & 0.69 \\
\hline Primary & $2179(89)$ & $1724(79)$ & $455(21)$ & \\
\hline Recurrent & $272(11)$ & $218(80)$ & $54(20)$ & \\
\hline \multicolumn{4}{|c|}{ Number of T1G3 tumors, $n(\%)$} & 0.95 \\
\hline Single & $1198(49)$ & 934 (78) & $264(22)$ & \\
\hline Multiple & $590(24)$ & $459(78)$ & $131(22)$ & \\
\hline \multicolumn{4}{|c|}{ Total number tumors (even not T1G3), $n(\%)$} & $<0.001$ \\
\hline Single & $964(39)$ & $823(85)$ & $141(15)$ & \\
\hline Multiple & $1365(56)$ & $1012(74)$ & $353(26)$ & \\
\hline \multicolumn{4}{|c|}{ T1G3 tumor size, $n(\%)$} & $<0.001$ \\
\hline$<3 \mathrm{~cm}$ & $1137(46)$ & $965(85)$ & $172(15)$ & \\
\hline$\geq 3 \mathrm{~cm}$ & $560(23)$ & $422(75)$ & $138(25)$ & \\
\hline \multicolumn{4}{|c|}{ Concomitant CIS, $n(\%)$} & $<0.001$ \\
\hline No & $1852(76)$ & $1504(81)$ & 348 (19) & \\
\hline Yes & $599(24)$ & $438(73)$ & $161(27)$ & \\
\hline \multicolumn{4}{|c|}{ BCG maintenance, $n(\%)$} & $<0.001$ \\
\hline No & $1515(62)$ & $1148(76)$ & $367(24)$ & \\
\hline Yes & $936(38)$ & $794(85)$ & $142(15)$ & \\
\hline
\end{tabular}

advanced T3/T4 disease and $72(21 \%)$ had lymph-node involvement at RC. There was no difference in the time to $\mathrm{T} 3 / \mathrm{T} 4$ or $\mathrm{N}+$ disease at cystectomy according to whether muscle-invasive disease was diagnosed before cystectomy (113 of 226 patients, $50 \%$ ) or only at cystectomy (61 of 111 patients, 55\%), HR $=1.19,95 \%$ CI $0.87-1.63$, $p=0.27$ (Fig. 2). Patients with tumors $\geq 3 \mathrm{~cm}$ or with multiple tumors (HR $=1.71,95 \%$ CI 1.22-2.41, $p=0.002$ and $\mathrm{HR}=1.79,95 \% \mathrm{CI} 1.25-2.56, p=0.001)$ and patients with tumors $\geq 3 \mathrm{~cm}$ or with concomitant CIS (HR $=1.79,95 \%$ CI $1.27-2.51, p=0.001$ and $\mathrm{HR}=1.53,95 \%$ CI $1.06-2.19$, $p=0.02$ ) had earlier T3 or T4 or $\mathrm{N}+$ cystectomies.

Overall, 196 (39\%) of the patients undergoing cystectomy died. Among the 113 (22\%) patients who died due to bladder cancer, 76 (67\%) progressed before RC, 25 (22\%) had a progression at the pathology specimen of RC, while only $12(11 \%)$ did not progress to muscle invasive before or at surgery. Time to bladder cancer-specific mortality
Table 2 Disease characteristics at cystectomy and survival according to the timing of cystectomy among the cohort of 509 T1G3 patients treated with BCG who underwent radical cystectomy

\begin{tabular}{|c|c|c|c|c|}
\hline Variables & Cyst, no prog & Prog $<$ Cyst & Prog $=$ Cyst & $p$ value \\
\hline $\begin{array}{l}\text { Number of } \\
\text { patients }\end{array}$ & $172(34)$ & $226(44)$ & $111(22)$ & \\
\hline \multicolumn{4}{|c|}{ Nodal status at cystectomy, $n(\%)$} & $<0.001$ \\
\hline No & $64(37)$ & $122(54)$ & $68(61)$ & \\
\hline $\mathrm{N}+$ & 0 & $48(21)$ & $24(22)$ & \\
\hline Missing & $108(63)$ & $56(25)$ & $19(17)$ & \\
\hline \multicolumn{4}{|c|}{ Survival status, $n(\%)$} & $<0.001$ \\
\hline Alive & $139(82)$ & $107(47)$ & $67(60)$ & \\
\hline Dead & $33(18)$ & $119(53)$ & $44(40)$ & \\
\hline \multicolumn{5}{|c|}{ Cause of death, $n(\%)$} \\
\hline Bladder cancer & $12(7)$ & $76(34)$ & $25(23)$ & \\
\hline $\begin{array}{l}\text { Other malig- } \\
\text { nancies }\end{array}$ & $3(2)$ & $13(6)$ & $8(7)$ & \\
\hline $\begin{array}{l}\text { Treatment } \\
\text { related }\end{array}$ & $2(1)$ & $8(4)$ & 0 & \\
\hline Chronic disease & $5(3)$ & $8(4)$ & $6(5)$ & \\
\hline Unknown & $11(6)$ & $14(6)$ & $5(4)$ & \\
\hline
\end{tabular}

according to the timing of the cystectomy is reported in Fig. 3.

In a Cox multivariable analysis including gender, the presence of extravesical disease at cystectomy, the presence of CIS at cystectomy and the timing of progression relative to cystectomy, patients with $\mathrm{T} 3 \mathrm{~T} 4$ or $\mathrm{N}+$ disease at radical cystectomy had a shorter time to death due to bladder cancer after cystectomy (HR $=4.38,95 \%$ CI 2.76-6.97, $p<0.001)$, as did patients with CIS at cystectomy ( $\mathrm{HR}=2.39,95 \%$ CI 1.59-3.59, $p<0.001)$. There was also a suggestion that patients who progressed prior to cystectomy had a shorter disease-specific survival after cystectomy than patients for whom the progression was only detected at cystectomy $(\mathrm{HR}=0.58,95 \%$ CI $0.36-0.93, p=0.024) .48 \%$ of the patients who progressed prior to cystectomy had a reTUR as compared to $39 \%$ of the patients who progressed at cystectomy. $71 \%$ of the patients who had a cystectomy but did not progress had a reTUR.

\section{Discussion}

In this retrospective study, we evaluated the impact of timing of RC in a large multicenter cohort of T1G3 BCa patients treated with BCG who finally underwent RC. Moreover, we evaluated predictors of RC, of extravesical disease at RC and of bladder cancer-specific survival after RC. We found that tumor size $>3 \mathrm{~cm}$ and the presence of concomitant CIS were the most important predictors of RC. Moreover, based on our findings, the concomitant presence of both of these 
Fig. 1 Time to cystectomy based on tumor size (greater than or equal to $3 \mathrm{~cm}$ ) and the presence of concomitant CIS among the cohort of T1G3 patients treated with BCG. GE greater than or equal to, $C I S$ concomitant carcinoma in situ. Time zero is the date of starting BCG $p<0.001$
Fig. 2 Time to T3/T4 or $\mathrm{N}+$ disease at cystectomy according to the timing of cystectomy (progression before cystectomy, progression at cystectomy) among the cohort of $\mathrm{T} 1 \mathrm{G} 3$ patients treated with BCG who finally underwent radical cystectomy. Prog $<$ Cyst progression before cystectomy, Prog $=$ Cyst progression at cystectomy. Time zero is the date of starting BCG $p=0.27$
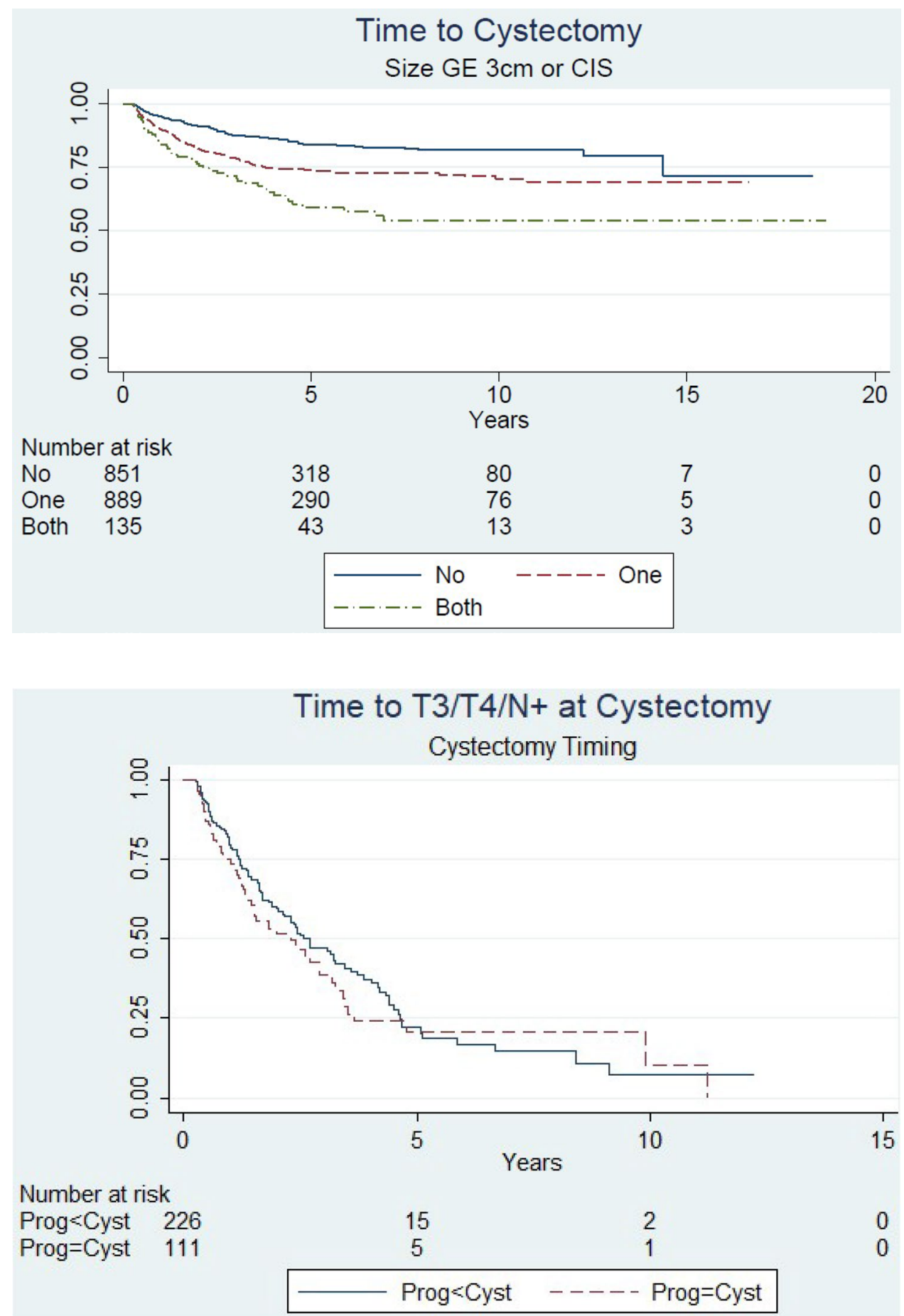

factors identified the patients who were the most likely to undergo surgery. Factors predicting the occurrence of RC in T1G3 patients have been rarely reported in literature. Actually, most published trials have investigated predictors of progression to muscle-invasive disease, reporting age, gender, tumor size, concomitant CIS and lympho-vascular invasion as the main factors [1, 2]. Independent of the risk of progression, understanding predictive factors of radical treatment will be helpful in the decision-making process and in patient counseling.

We showed that tumor size $>3 \mathrm{~cm}$, concomitant CIS and the presence of multiple tumors were the most important predictors of extravesical disease at $\mathrm{RC}$ in this large series of T1G3 treated with BCG. Since locally advanced disease and lymph-node involvement are strictly related to unfavorable oncological and survival outcomes [12, 13], a more 


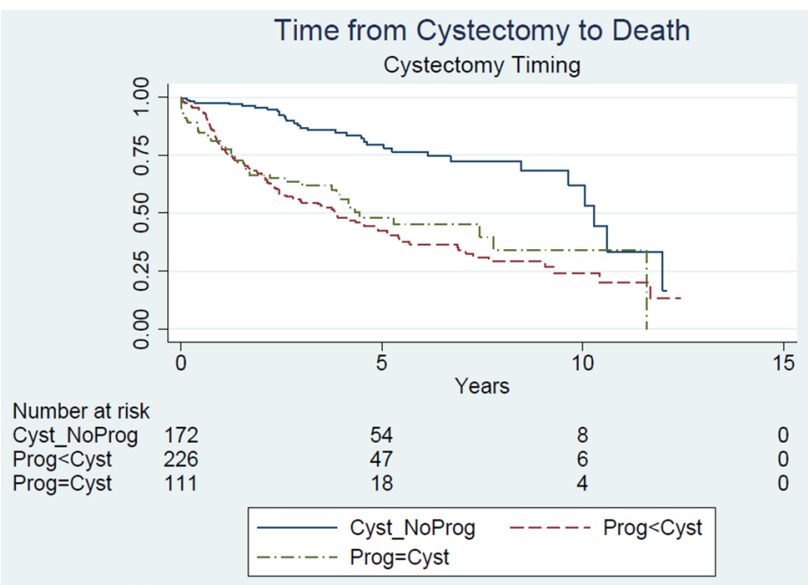

Fig. 3 Kaplan Meier curve according to time cancer-specific death based on the timing of cystectomy (no progression, progression before cystectomy, progression at cystectomy) among the cohort of T1G3 patients treated with BCG who finally underwent radical cystectomy. Cyst_NoProg no progression, Prog<Cyst progression before cystectomy, Prog=Cyst progression at cystectomy

aggressive strategy should be taken into consideration in T1G3 patients presenting these characteristics at diagnosis.

Our findings partially reflect the published literature. Previously, Palou et al. in a single-institution retrospective analysis identified the presence of CIS in the prostatic urethra and female gender as prognostic factors for time to recurrence, progression, and cancer-specific mortality in T1G3 patients treated with induction BCG [14]. Similarly, Shariat et al. reported the presence of concomitant CIS as the only pre-cystectomy factor able to predict disease recurrence after surgery and mortality [15]. Finally, in one of the largest meta-analyses performed in T1 high-grade NMIBC patients, depth of invasion into lamina propria, the presence of lympho-vascular invasion and the presence of CIS were found to be the main predictors of intravesical recurrence, progression and cancer-specific survival [1].

We found that RC patients who did not experience progression to muscle-invasive disease experienced better disease-specific survival outcomes compared to those who progressed during follow-up before receiving surgery or to those who were diagnosed with muscle-invasive tumor at RC. The latter represent the cohort of patients who were upstaged at RC (in our study $22 \%$ ).

Upstaging to muscle-invasive disease is, unfortunately, a common finding in NMIBC patients, especially in T1G3 tumors. Several retrospective studies reported upstaging rates at final pathology varying from 20 to $68 \%$ depending on the series [16-18].

Finally, we found, as expected, that patients with T3T4 or $\mathrm{N}+$ disease at radical cystectomy had a shorter time to death due to bladder cancer after cystectomy, as did patients with CIS at cystectomy. In addition, our data also suggest that patients who progress prior to cystectomy have a shorter disease-specific survival after cystectomy than patients for whom the progression is only detected at cystectomy.

Our results are consistent with the literature and reinforce the need for the surgical treatment of T1G3 patients before progression to muscle-invasive disease. Actually, it is well known that tumor progression is responsible for an unfavorable prognosis, even when treated with RC [19] and that patients undergoing early cystectomy with pathological $\mathrm{T} 1$ disease have a significantly higher 10-year disease-free survival than those with clinical T1 disease who are upstaged on pathological analysis (92\% vs 64\%) [20, 21]. Moreover, early cystectomy seems to provide better 5 - and 10-year cancer-specific survival rates compared to deferred surgery $[7,10]$. However, these advantages must be weighed against the morbidity/mortality of RC and the risk of overtreatment.

Therefore, we believe that our findings have a clinical impact since they can help to identify those patients who, despite an initial conservative strategy, are more likely to finally receive $\mathrm{RC}$ and also to experience worse oncological outcomes. This, in combination with the impact of the timing of RC on outcomes, reinforces the need to quickly and timely select patients for early $\mathrm{RC}$ without uselessly delaying a potentially life-saving treatment.

Despite its strengths, our study is not devoid of limitations, mainly due to its retrospective nature. First, disease status just before cystectomy, some pathological characteristics (like $\mathrm{T}$ substaging and lymphovascular invasion) and the use of neoadjuvant chemotherapy were not available for all patients included in the study. Moreover, as previously reported [2], accuracy in reporting prognostic factors such as tumor size and number of tumors suffered from missing data and lack of standardized assessment. This lack of standardization is due to both the retrospective nature of the study and the large recruitment interval. Moreover, a second trans-urethral resection of the bladder (TUR-B) was performed in only 258 (51\%) of the 509 patients and this could have limited the conclusions. No central pathology review was performed. Finally, this cohort may represent a selected population of T1G3 patients with initial favorable characteristics since all of them received BCG as first treatment. However, this could also be a strength of our work since it makes our cohort more homogeneous and reflects more the everyday clinical practice, making our conclusions more generalizable and widely applicable.

\section{Conclusions}

A non-negligible rate of T1G3 BCa patients initially treated with BCG will finally undergo RC. Patients treated with RC before experiencing tumor progression to muscle-invasive disease harbor better oncological and survival outcomes 
compared to those who progressed before $\mathrm{RC}$ and to those upstaged at surgery. Tumor size and concomitant CIS at diagnosis are the main predictors of surgical treatment while tumor size, CIS and tumor multiplicity are associated with extravesical disease at surgery. Identification of predictive factors can be helpful during patient counseling and in the treatment decision process to select patients for early RC.

Acknowledgements The authors declare that the development of the manuscript was not supported by an honorarium, a grant, or any other sources of support, including sponsorship or any material sources of support.

Author contributions Study concept and design: PG, RS; Acquisition of data: FP, SJ, VS, JP, SL, SS, BR, AW, AG, RC, AB, MB, VS, PUM, JI, NM, JB, RM, TC, EC, PA, JV, RB, GD, SFS, EX, RJK; Analysis and interpretation of data: RS, FS, PF; Drafting of the manuscript: $\mathrm{PF}, \mathrm{FS}$; Critical revision of the manuscript for important intellectual content: RS, SJ, VS, SL, SS, BR, AW, AG, RC, AB, MB, VS, PUM, JI, NM, JB, RM, TC, EC, PA, JV, RB, GD, SFS, EX, RJK, PJ; Statistical analysis: RS; Administrative, technical, or material support: FP; Supervision: RS, PG.

\section{Compliance with ethical standards}

Research involving human participants and/or animals The study has been performed in accordance with the ethical standards laid down in the 1964 Declaration of Helsinki and its later amendments.

Informed consent For this type of study, informed consent is not required.

Conflict of interest The authors declare that they have no conflict of interest.

\section{References}

1. Martin-Doyle W, Leow JJ, Orsola A, Chang SL, Bellmunt J (2015) Improving selection criteria for early cystectomy in high-grade T1 bladder cancer: a meta-analysis of 15,215 patients. J Clin Oncol 33:643-650. https://doi.org/10.1200/JCO.2014.57.6967

2. Gontero P, Sylvester R, Pisano F, Joniau S, Vander Eeckt K, Serretta V et al (2015) Prognostic factors and risk groups in T1G3 non-muscle-invasive bladder cancer patients initially treated with Bacillus Calmette-Guerin: results of a retrospective multicenter study of 2451 patients. Eur Urol 67:74-82. https://doi. org/10.1016/j.eururo.2014.06.040

3. Cookson MS, Herr HW, Zhang ZF, Soloway S, Sogani PC, Fair WR (1997) The treated natural history of high risk superficial bladder cancer: 15-year outcome. J Urol 158:62-67. https://doi. org/10.1097/00005392-199707000-00017

4. Segal R, Yafi FA, Brimo F, Tanguay S, Aprikian A, Kassouf W (2012) Prognostic factors and outcome in patients with T1 highgrade bladder cancer: can we identify patients for early cystectomy? BJU Int 109:1026-1030. https://doi.org/10.1111/j.1464410X.2011.10462.x

5. Shahin O, Thalmann GN, Rentsch C, Mazzucchelli L, Studer UE (2003) A retrospective analysis of 153 patients treated with or without intravesical Bacillus Calmette-Guerin for primary stage $\mathrm{T} 1$ grade 3 bladder cancer: recurrence, progression and survival. J Urol 169:96-100. https://doi.org/10.1097/01. ju.0000035543.69161.58

6. Kim HS, Kim M, Jeong CW, Kwak C, Kim HH, Ku JH (2014) Presence of lymphovascular invasion in urothelial bladder cancer specimens after transurethral resections correlates with risk of upstaging and survival: a systematic review and meta-analysis. Urol Oncol 32:1191-1199. https://doi.org/10.1016/j.urolo nc.2014.05.008

7. Denzinger S, Fritsche HM, Otto W, Blana A, Wieland WF, Burger M (2008) Early versus deferred cystectomy for initial high-risk pT1G3 urothelial carcinoma of the bladder: do risk factors Define feasibility of bladder-sparing approach? Eur Urol 53:146-152. https://doi.org/10.1016/j.eururo.2007.06.030

8. De Berardinis E, Busetto GM, Antonini G, Giovannone R, Gentile V (2011) T1G3 high-risk NMIBC (non-muscle invasive bladder cancer): conservative treatment versus immediate cystectomy. Int Urol Nephrol 43:1047-1057. https://doi. org/10.1007/s11255-011-9941-x

9. Babjuk M, Burger M, Zigeuner R, Shariat SF, van Rhijn BWG, Compérat E et al (2017) EAU guidelines on non-muscle-invasive urothelial carcinoma of the bladder: update 2016. Eur Urol 71(3):447-461. https://doi.org/10.1016/j.eururo.2016.05.041

10. Hautmann RE, Volkmer BG, Gust K (2009) Quantification of the survival benefit of early versus deferred cystectomy in highrisk non-muscle invasive bladder cancer (T1 G3). World J Urol 27:347-351. https://doi.org/10.1007/s00345-009-0402-4

11. Herr HW, Faulkner JR, Grossman HB, Natale RB, deVere WR, Sarosdy MF et al (2004) Surgical factors influence bladder cancer outcomes: a cooperative group report. J Clin Oncol 22:2781-2789. https://doi.org/10.1200/JCO.2004.11.024

12. Babjuk M, Burger M, Zigeuner R, Shariat SF, van Rhijn BWG, Compérat $\mathrm{E}$ et al (2013) EAU guidelines on non-muscle-invasive urothelial carcinoma of the bladder: update 2013. Eur Urol 64:639-653. https://doi.org/10.1016/j.eururo.2013.06.003

13. Zehnder P, Studer UE, Skinner EC, Thalmann GN, Miranda G, Roth B et al (2013) Unaltered oncological outcomes of radical cystectomy with extended lymphadenectomy over three decades. BJU Int 112:E51-E58. https://doi.org/10.1111/bju.12215

14. Shariat SF, Karakiewicz PI, Palapattu GS, Lotan Y, Rogers CG, Amiel GE et al (2006) Outcomes of radical cystectomy for transitional cell carcinoma of the bladder: a contemporary series from the Bladder Cancer Research Consortium. J Urol 176:2414-2422. https://doi.org/10.1016/j.juro.2006.08.004 (discussion 2422)

15. Palou J, Sylvester RJ, Faba OR, Parada R, Peña JA, Algaba $F$ et al (2012) Female gender and carcinoma in situ in the prostatic urethra are prognostic factors for recurrence, progression, and disease-specific mortality in T1G3 bladder cancer patients treated with Bacillus Calmette-Guérin. Eur Urol 62:118-125. https://doi. org/10.1016/j.eururo.2011.10.029

16. Gupta A, Lotan Y, Bastian PJ, Palapattu GS, Karakiewicz PI, Raj GV et al (2008) Outcomes of patients with clinical T1 grade 3 urothelial cell bladder carcinoma treated with radical cystectomy. Urology 71:302-307. https://doi.org/10.1016/j.urolo gy.2007.10.041

17. Ficarra V, Dalpiaz O, Alrabi N, Novara G, Galfano A, Artibani W (2005) Correlation between clinical and pathological staging in a series of radical cystectomies for bladder carcinoma. BJU Int 95:786-790. https://doi.org/10.1111/j.1464-410X.2005.05401.x

18. Thalmann GN, Markwalder R, Shahin O, Burkhard FC, Hochreiter WW, Studer UE (2004) Primary T1G3 bladder cancer: organ preserving approach or immediate cystectomy? J Urol 172:70-75. https://doi.org/10.1097/01.ju.0000132129.87598.3b

19. Fritsche H-M, Burger M, Svatek RS, Jeldres C, Karakiewicz PI, Novara G et al (2010) Characteristics and outcomes of patients with clinical T1 grade 3 urothelial carcinoma treated with 
radical cystectomy: results from an international cohort. Eur Urol 57:300-309. https://doi.org/10.1016/j.eururo.2009.09.024

20. van den Bosch S, Alfred WJ (2011) Long-term cancer-specific survival in patients with high-risk, non-muscle-invasive bladder cancer and tumour progression: a systematic review. Eur Urol 60:493-500. https://doi.org/10.1016/j.eururo.2011.05.045
21. Bianco FJ, Justa D, Grignon DJ, Sakr WA, Pontes JE, Wood DP (2004) Management of clinical T1 bladder transitional cell carcinoma by radical cystectomy. Urol Oncol 22:290-294. https://doi. org/10.1016/S1078-1439(03)00144-3

\section{Affiliations}

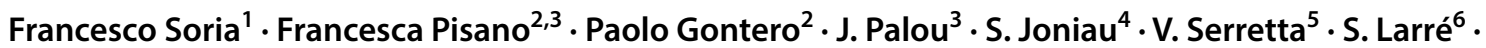

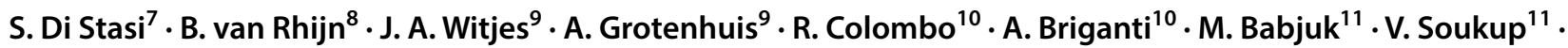

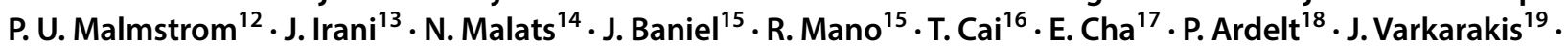

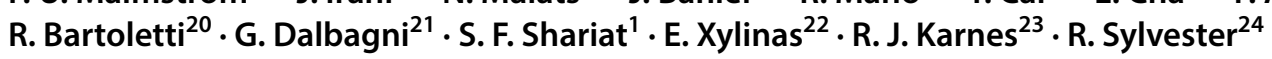

1 Department of Urology, Medical University of Vienna, Vienna, Austria

2 Città della Salute e della Scienza di Torino, University of Studies of Turin, Turin, Italy

3 Department of Urology, Fundacio Puigvert, University of Barcelona, Barcelona, Spain

4 Oncologic and Reconstructive Urology, Department of Urology, University Hospitals Leuven, Louvain, Belgium

5 Department of Surgical, Oncological and Stomatological Sciences, University of Palermo, Palermo, Italy

6 Department of Surgical Science, John Radcliffe Hospital, University of Oxford, Oxford, UK

7 Policlinico Tor Vergata-University of Rome, Rome, Italy

8 Department of Urology, Netherlands Cancer Institute-Antoni van Leeuwenhoek Hospital, Amsterdam, The Netherlands

9 Department of Urology, Radboud University Nijmegen Medical Centre, Nijmegen, The Netherlands

10 Dipartimento di Urologia, Università Vita-Salute. Ospedale S. Raffaele, Milan, Italy

11 Department of Urology, Motol Hospital, University of Praha, Prague, Czech Republic

12 Department of Urology, Academic Hospital, Uppsala University, Uppsala, Sweden
13 Department of Urology, CHU de Bicêtre, 78, rue du Général Leclerc, 94270 Le Kremlin-Bicêtre, France

14 Genetic and Molecular Epidemiology Group, Spanish National Cancer Research Centre (CNIO), Madrid, Spain

15 Department of Urology, Rabin Medical Centre, Tel Aviv, Israel

16 Department of Urology, Santa Chiara Hospital, Trento, Italy

17 Department of Urology, Weill Medical College of Cornell University in New York City, New York, NY, USA

18 Facharzt fur Urologie, Abteilung fur Urologie, Chirurgische Universitats klinik, Freiburg, Germany

19 Department of Urology, Sismanoglio Hospital, University of Athens, Athens, Greece

20 Department of Experimental and Clinical Medicine, University of Florence, Florence, Italy

21 Department of Urology, Memorial Sloan Kettering Cancer Center, New York, NY, USA

22 Department of Urology, Cochin Hospital, Paris, France

23 Department of Urology, Mayo Clinic, Rochester, MN, USA

24 Formerly Department of Biostatistics, EORTC Headquarters, Brussels, Belgium 